\title{
Polarization sensitive optical biopsy with diffusely reflected polarized light
}

\author{
Alexander Bykov*, Alexey Popov \\ Optoelectronics and Measurement Techniques Laboratory, University of Oulu, P.O. Box 4500, 90014 Oulu, Finland
}

Tatiana Novikova

LPICM, CNRS, Ecole Polytechnique, Université Paris-Saclay, 91128 Palaiseau, France

Alexander Doronin

Department of Computer Science, Yale University, P.O. Box 208285, New Haven, CT 06520-8285, USA

Igor Meglinski

Optoelectronics and Measurement Techniques Laboratory, University of Oulu, P.O. Box 4500, 90014 Oulu, Finland

* Correspondence e-mail address: bykov@ee.oulu.fi

\begin{abstract}
We report the results of comparative non-invasive screening of cancerous and noncancerous tissue samples in vitro with two major polarization-based diagnostic modalities utilizing Jones vector and Mueller matrix formalisms. The technical aspects of both experimental approaches, peculiarities of data analysis, detected signal formation, opportunities for clinical use, future perspectives and developments are presented and discussed.

OCIS codes: (170.3660) Light propagation in tissues; (170.6935) Tissue characterization; (260.2130); Ellipsometry and polarimetry; (260.5430) Polarization; (290.5855) Scattering, polarization.
\end{abstract}

\section{Introduction}

Early detection of cancer is extremely important for the successful treatment. However, during early cancer onset it is quite difficult for clinicians and pathologists to differentiate between tissues that could be neoplastic versus normal tissue that is unlikely to become neoplastic. Currently, the gold standard and most widely used methodology for precise cancer diagnosis is histological analysis that utilizes exhaustive microscopy investigation. However, despite best laboratory practices the rate of conclusive diagnosis by histological analysis for a range of cancers, including cervical, prostate, bladder, skin and oral cancer, is only 65-75\%. For example, only $70 \%$ of prostate cancer cases are detected during the initial biopsy.

\section{Jones vector based experimental approach}

We introduce a development of experimental optical system (Fig. 1) for noncontact diagnostics of biological tissues by using diffusively reflected polarized light. In the experiment the vertical polarized light from a laser diode $\left(640 \mathrm{~nm}, 40 \mathrm{~mW}\right.$, BioRay Laser, Coherent, USA) is directed towards the tissue sample at $55^{\circ}$ from the normal. The light is then altered by a quarter wave plate into a state of right circular polarized and/or right elliptically polarized, and focused onto the sample by a lens. Incident linear, circularly or elliptically polarized light is propagated through the tissue sample and undergoes multiple scattering events before being collected by the detector.

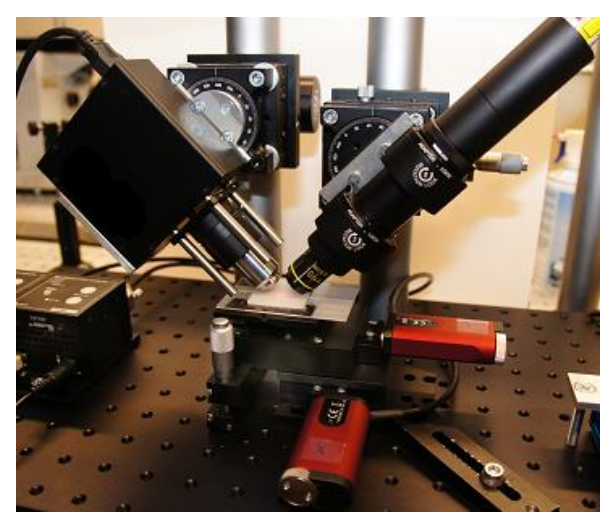

Fig.1. The experimental setup specially developed for automatic screening of cancerous and non-cancerous tissue samples in vitro. The polarized laser light is focused onto the surface of tissue sample. Diffusively back-scattered optical radiation is collected at a distance $\sim 1 \mathrm{~mm}$ away from the focus point and analysed utilizing conventional optical polarimeter.

Diffusely backscattered polarized light is collected at a distance $d$ from the focus point and is then passed through a conventional polarimeter (PAX5710VIS-T, Thorlabs, USA) to measure degree of polarization. The 
source detector separation as well as the angle of detection can be varied to influence the sampling volume. To assess the optimal source-detector separation and angles of incidence and detection, the Monte Carlo code developed in house has been extensively used for the actual parameters of the experimental system [1,2].

The multiple measurements have been performed with the developed experimental system (see Fig.1) utilizing scanning approach on a human lung metastasis of basal squamous cell carcinoma embedded in paraffin wax. These samples had a variety of tissue structures present, including both healthy and cancerous tissue previously classified by the pathologist. To visualize and compare the polarization state of the detected radiation the Poincare sphere was used as suggested in Ref. [3]. The cancerous and healthy tissue samples have shown that their scattered polarization states are clearly distinguishable from one another. It is noticeable that the polarization state of the diffusively backscattered light from the cancerous samples is located mostly on the upper regions of the northern hemisphere, while the healthy tissues correspond to lower latitudes. The proposed approach has great potential to be used as a new optical biopsy tool helping the pathologists in routine day-today diagnostic decisions.

\section{Mueller matrix based experiment}

To validate the developed experimental system the alternative Muller matrix based approach [4] has been applied. Traditionally interaction of polarized light with the sample is described by the Mueller matrix that plays a role of polarimetric transfer function of the medium. It is a 4x4 element matrix that relates the output and the input light polarization. Recent studies have shown that the Mueller matrix is an information-rich tissue signature containing the structural and optical properties of complex tissues, and has been widely applied to biomedical diagnosis including cancer detection [4,5]. After the measurements, that often are challenging, the Mueller matrix of the medium should be further analyzed via various decomposition methods. Traditional and most successful decomposition approach was introduced by Lu and Chipman [6]. In this approach, the turbid media is represented as a sequence of depolarizer, retarder and diattenuator that allows extracting the corresponding properties. Typical setup for the Mueller matrix measurement (imaging Mueller polarimeter) is shown in Fig. 2a.
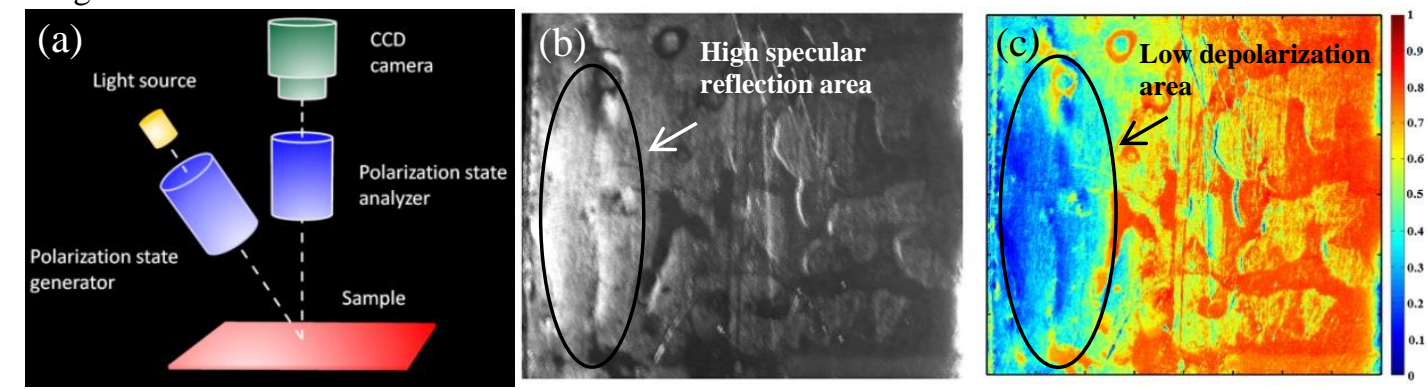

Fig. 2. (a) - Schematic presentation of Mueller polarimeter. (b) - image of sample surface. (c) - Depolarization map of the sample. Field of view is $3 \times 3.5 \mathrm{~cm}$.

\section{Summary}

In current report we present the results of comparative non-invasive screening of cancerous and noncancerous tissue samples in vitro with two major polarization-based diagnostic techniques utilizing Jones vector and Mueller matrix formalisms. The technical aspects of both experimental approaches, peculiarities of data analysis and detected signal formation as well as opportunities for clinical use, future perspectives and developments are outlined and discussed.

\section{References}

[1] A. Doronin, I. Meglinski, "Online object oriented Monte Carlo computational tool for the needs of biomedical optics", Biomed. Opt. Exp., 2, 2461-2469 (2011).

[2] V.L. Kuzmin, I. Meglinski, "Coherent effects of multiple scattering for scalar and electromagnetic fields: Monte-Carlo simulation and Milne-like solutions", Opt. Communications, 273, 307-310 (2007).

[3] B. Kunnen, C. Macdonald, A. Doronin, S. Jacques, M. Eccles, I. Meglinski, "Application of circularly polarized light for noninvasive diagnosis of cancerous tissues and turbid tissue-like scattering media”, J. Biophoton., 8(4), 317-323 (2015).

[4] A. Pierangelo, S. Manhas, A. Benali, C. Fallet, J.-L. Totobenazara, M.-R. Antonelli, T. Novikova, B. Gayet, A. De Martino, P. Validire, "Multispectral Mueller polarimetric imaging detecting residual cancer and cancer regression after neoadjuvant treatment for colorectal carcinomas", J. Biomed. Opt., 18(4), 046014 (2013).

[5] N. Agarwal, J. Yoon, E. Garcia-Caurel, T. Novikova, J.-C. Vanel, A. Pierangelo, A. Bykov, A. Popov, I. Meglinski, R. Ossikovski, "Spatial evolution of depolarization in homogeneous turbid media within the differential Mueller matrix formalism", Opt. Lett., 40(23), 5634-5637 (2015).

[6] S.-Y. Lu, R.A. Chipman, "Interpretation of Mueller matrices based on polar decomposition”, J. Opt. Soc. Am. A, 13(5), 1106-1113 (1996). 